\title{
El influencer literario a través de YouTube: análisis de la estrategia comunicativa
}

\section{The literary influencer through YouTube: analysis of its communication strategy}

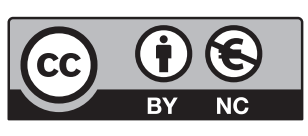

\section{Marilé Pretel Jiménez}

Universidad CEU San Pablo Facultad de Humanidades y Ciencias de la Comunicación

Doctora en Ciencias de la Información. Profesora de Gestión de marcas. Vicedecana de la Facultad de Humanidades y Ciencias de la Comunicación, Universidad CEU San Pablo.

mapretel.fhm@ceu.es

orcid.org/0000-0001-6775-047X

\section{Belinda de Frutos}

Universidad de Valladolid Facultad de Ciencias Sociales, Jurídicas y de la Comunicación

Doctora en Psicología por la Universidad Autónoma de Madrid. Desarrolló su doctorado en el Departamento de Psicología Social y Metodología de la Universidad Autónoma de Madrid y en Skidmore University College (USA). Profesora Contratada Doctora en la Universidad de Valladolid en la titulación de Publicidad y Relaciones Públicas, trabajó en la Universidad San Pablo CEU y en el IE University. Ha desarrollado su actividad profesional en la investigación de mercados y opinión.

belinda.defrutos@hmca.uva.es

orcid.org/0000-0002-9391-8835

\section{María Sánchez-Valle}

Universidad CEU San Pablo Facultad de Humanidades y Ciencias de la Comunicación

Doctora en Ciencias de la Información por la Universidad Pontificia de Salamanca. Profesora adjunta del Grado en Publicidad y Relaciones Públicas de la Universidad CEU San Pablo y directora del Máster en Relaciones Públicas y Organización de Eventos. Es miembro de dos proyectos de investigación que estudian la comunicación dirigida a públicos vulnerables. mvalle.fhum@ceu.es orcid.org/0000-0003-1497-2938

Fecha de recepción: 2 de febrero de 2018 / Aceptación: 28 de noviembre de 2018 


\section{Resumen}

Surge en el entorno digital la figura del booktuber. El nuevo prescriptor digital literario en la red. El objetivo de este trabajo busca conocer la estrategia de comunicación de esta nueva figura. Identificando los 10 booktubers más relevantes en España, se analiza la estructura organizativa de la información, qué contenido consigue más visitas e interacción, así como la estrategia en sus redes sociales. Los resultados muestran que se puede identificar una estructura de contenidos, e identifica los más relevantes, pudiéndose establecer un primer marco de carácter conceptual sobre estrategias de comunicación que impacten en un público joven en donde se consiga fomentar el gusto por la actividad lectora.

\section{PALABRAS CLAVE}

Booktubers, jóvenes, influencers, lectura, redes sociales.

\section{Abstract}

A new literary prescriber has recently emerged in the digital environment, the BookTuber. The objective of this article is to determine the communication strategy of this new figure. Through the identification of 10 relevant BookTubers in Spain, we analyze the organizational structure of the information, their social network strategy and the video content that get more visits and interaction. The results show that a structure of content that follows a similar pattern can be identified in the studied cases. The results also identify what contents are more relevant, allowing to establish a first conceptual framework on the content and communication strategies in social networks to impact a young target audience and promote the taste for reading.

\section{KEYWORDS}

BookTubers, young, reading, internet social networking.

\section{INTRODUCCIÓN}

Hoy en día, las redes sociales se han consolidado como el medio preferido por los jóvenes para relacionarse, mantenerse informados sobre la actualidad y compartir o generar contenido. Muchas son las fuentes que confirman los datos de penetración de las redes sociales y de la frecuencia de interacción de este público en el entorno digital (AIMC; IAB; The Cocktail Analysis, 2015).

En este sentido Ravettino (2015), apunta que las TIC y específicamente el uso de Internet está dando lugar a nuevas prácticas y acercamientos al mundo de la literatura y al de la lectura en particular. En la misma línea, Urresti (2008) hace referencia a estos espacios virtuales donde se establece un vínculo de conexión entre los jóvenes y en donde se comparte y se genera contenido. A este fenómeno lo denomina "ciberculturas juveniles".

Es en este universo digital donde se ha consolidado una de las comunidades con mayor número de público: YouTube, con más de mil millones de usuarios. Y es precisamente al amparo de esta red donde aparece una nueva figura generadora de contenido, los booktubers, personas de diferentes edades, en su mayoría jóvenes lectores que difunden en la Red comentarios sobre libros. Esta nueva figura se erige como un posible nuevo líder de opinión que tiene como finalidad principal compartir el placer por la lectura con un lenguaje cercano y llano. La mayoría de ellos son jóvenes, mayoritariamente adolescentes que hablan a cámara dando una opinión personal sobre los libros que leen. 
El fenómeno estudiado adquiere doble relevancia como objeto de análisis; en primer lugar, por la importancia que desde el sector de la educación y de la administración pública tiene el apoyo del fomento de la lectura, aspecto clave en la formación de generaciones futuras. Y, en segundo lugar, por la realidad social ante la que nos encontramos que nos revela cambios en el acceso a la lectura en las generaciones de jóvenes españoles. Según datos del Ministerio de Cultura, apenas 4.500 jóvenes entre los 15 y 24 años leyeron un libro en el último año (MECD, 2015).

El objetivo principal y específico del trabajo que se describe a continuación es ofrecer un primer acercamiento a la figura emergente de un nuevo influenciador: el booktuber como nuevo prescriptor literario y como figura posiblemente clave en el desarrollo de una estrategia de comunicación que busque el fomento de la lectura. Se trata, por tanto, de profundizar en el análisis de la estructura y el contenido de este nuevo perfil como figura que promueve el hábito de la lectura entre un público que precisamente en la adolescencia suele manifestar un desencuentro con el hábito lector (Pindado, 2003).

\section{MARCO TEÓRICO}

\subsection{LA FIGURA DEL INFLUENCER EN EL CONTEXTO DIGITAL}

La utilización de las redes sociales por parte de algunos usuarios como entorno donde poder compartir contenidos propios de interés para un gran número de seguidores ha dado lugar a la figura del influencer. Detrás de este fenómeno hay un proceso de influencia social que puede ser interpretado desde el modelo clásico de comunicación de Katz y Lazerfield (1966) so- bre los líderes de opinión, pero que se debería adaptar a un nuevo escenario. Mason, Corren y Smith (2007) plantean una redefinición de los procesos de influencia interpersonal teniendo en cuenta el contexto en el que se producen y tomando una perspectiva temporal amplia. Así, los influencers tendrían como escenario las redes sociales en Internet, un espacio muy abierto comparado con los espacios de relación interpersonales y con muchas más posibilidades de interconexión personal. Al mismo tiempo emergen procesos de influencia interpersonal como la similitud percibida, la credibilidad o la autoridad de la fuente. Además, habría que añadir los fenómenos propios de las redes a través de Internet en las que emergen procesos de influencia a través de los individuos más cercanos o afines, cómo las redes se estructuran en torno a grupos con intereses afines y la validación social de la fuente a través del número de seguidores.

La aparición de una comunidad entorno al mundo literario donde se comentan libros en la plataforma YouTube es lo que se ha denominado booktuber, puede estudiarse desde esta perspectiva de influencia, como un caso particular de influencer. El alcance de la influencia interpersonal a través de las redes sociales viene avalado por la IAB (2015). Según sus últimos datos aportados, el 36\% de los españoles declara que utiliza la red para seguir a influencers. Este uso ocupa el tercer lugar en el ranking de actividades realizadas en Internet.

El fenómeno del booktuber tiene su origen en Estados Unidos. Mientras unas fuentes sitúan la aparición de estos usuarios en el año 2012 (Bluemagazine, 2016), otros autores como Goldfield (2015) consideran que el inicio se encuentra en el video blog denominado "Vlog brother" creado por dos hermanos estadounidenses y que en la actualidad tiene 3 millones de suscriptores. A partir de 2013 se extiende 
principalmente en México seguido de Argentina, Chile, Perú y Colombia y es en 2014 cuando empiezan a surgir las primeras figuras en España. Estos influencers literarios realizan reseñas sobre libros en sus canales de Youtube y lo comparten con los demás. La mayoría de los perfiles estudiados cumplen los siguientes criterios: en su mayoría son mujeres, adolescentes, leen libros de temáticas dirigidas a su edad, casi todas han hecho alguna reseña sobre los libros de la saga de Harry Potter. A la hora de producir el contenido, ellas mismas se graban un video en el que realizan un comentario sobre los libros que leen. Suelen tener un dominio multimedia alto y el contenido suele ser divertido y adictivo.

\subsection{JÓVENES, LECTURA Y REDES SOCIALES}

La lectura siempre ha sido un ejercicio tan natural en adultos que tendemos a olvidar el tiempo que nos ha llevado adquirir este hábito. También se tiende a creer que el hábito lector se desarrolla siempre de la misma manera sin tener en cuenta los cambios que se están produciendo dado el desarrollo tecnológico en el que nos vemos inmersos (Vandendorpe, 2011).

Junto con esta creencia se une la dinámica del mercado literario, que se encuentra en un momento de expansión. La publicación de obras crece, sin embargo, este fenómeno no ha ido acompañado de un movimiento similar en el número de lecturas promedio por persona. Se publican exponencialmente más libros de lo que crece la actividad lectora. Podría afirmarse que este fenómeno viene dado por la aparición de nuevos medios de expresión y espacios más eficaces desde el punto de vista comunicativo (Cordón, 2016).

Además, existen diversas fuentes de carácter público y privado que recogen datos relaciona- dos con la aparición de estos nuevos espacios digitales, y analizan la participación de los jóvenes españoles en las redes sociales, que confirman la dimensión del fenómeno interactivo en este sector poblacional. Datos que nos ayudarán a entender el auge que figuras como el booktuber tienen en el medio digital.

Los segmentos de población entre los 16 y los 24 años y entre los 25 y los 34 años son los usuarios más frecuentes de Internet con un $96,2 \%$ y un $89,9 \%$, respectivamente. Las cifras de uso de redes sociales son aún más elevadas en los mismos segmentos pasando a 98,5\% en jóvenes de 16 a 24 años y 96\% en usuarios de entre 25 y 34 años (ONTSI, 2015).

Según The Cocktail Analysis (2015) la penetración de usuarios internautas en redes sociales se mantiene estable desde el 2011. El 90\% de ellos mantiene al menos un perfil activo en alguna red social y de media cada usuario mantiene 2,3 cuentas activas. Otro aspecto de interés que aporta el estudio es el cambio que se está produciendo en la interacción entre el usuario y la red social donde se impone un modelo más inmediato, frecuente y poco profundo. Reflejo de ello es el uso de iconos y de las acciones de hacer retuits o dar un like / favorito, que han ganado en percepción de valor para el usuario junto al consumo cada vez mayor de contenidos audiovisuales. En este sentido cabe destacar la utilización universal de YouTube como red social que se ha convertido en plataforma satélite que proporciona contenidos al resto de redes sociales.

Si analizamos la actividad de estos jóvenes en la red (ONTSI, 2015) destacan las siguientes: participar en redes sociales, descarga de software, consulta de wikis y búsquedas relacionadas con educación o formación.

Otro aspecto importante en la comprensión de qué hace que una publicación resulte más 
atractiva que otra para este público, es aquel contenido que sea percibido por ellos como auténtico (43\%), relevante (32\%) y transparente (23\%) (Elite Daily's; Millennial Branding, 2015).

Todos estos datos vienen a corroborar que las redes sociales se han convertido en un espacio activo de consumo de contenido y encuentro, donde los usuarios generan, comparten y comentan temas frecuentemente. Dentro de este perfil es interesante ver cómo se puede distinguir entre dos tipos de comportamiento. El comportamiento de un usuario proactivo y el de uno pasivo. Entendiendo por activo el que escribe al menos un post todas las semanas, comenta o responde a otros, comparte links, sube fotos, etc.; mientras que el pasivo se dedica a revisar correos, ver videos, escucha música, etc.

\section{OBJETIVOS}

Estudios recientes sobre el fenómeno de este nuevo perfil influenciador han identificado aspectos formales sobre el uso del lenguaje utilizado en el discurso crítico sobre el contenido literario de las lecturas que recomiendan. En este sentido cabe destacar el estudio realizado por Beatriz Domínguez (Domínguez, 2016) donde entre otros aspectos, tanto positivos como negativos, resaltan algunos tales como el lenguaje cercano tal y como se estuvieran dirigiendo a un amigo, el uso de frases comodines, la falta de profundidad en el propio análisis, el dominio del lenguaje corporal frente a la cámara, etc. Aspectos importantes que nos ayudan a comprender los aspectos formales del fenómeno de influencia y conexión en los jóvenes, pero que dejan a un lado el análisis de otras variables que complementan el conocimiento sobre el éxito de determinados contenidos frente a otros.
Esta investigación se focaliza precisamente en el análisis de estas variables atendiendo a un objetivo principal, conocer la estrategia de contenidos desarrollada por estos booktubers teniendo en cuenta la estructura organizativa.

El estudio está limitado a los booktubers que editan sus contenidos en castellano y que son los más populares en el territorio español.

\section{METOdOLOGÍA}

Para la realización de este estudio descriptivo sobre la estructura y el contenido que genera este nuevo prescriptor literario se utilizó un muestreo no probabilístico delimitando el universo de estudio a los casos más relevantes en España. El estudio se ciñó a los 10 booktubers con mayor número de suscriptores en sus canales que, hasta el momento de la finalización del campo, tenían estos influencers. El periodo de análisis abarca desde noviembre de 2016 a enero 2017. Para el análisis de contenido se revisaron una media de entre 50/60 vídeos en cada canal.

Los objetivos que buscamos en este trabajo giran en torno a los siguientes puntos descritos:

- Análisis de cómo estructuran los contenidos en su canal

- Detectar cuáles son las redes enlazadas a sus canales de YouTube

- Realizar un análisis de contenido sobre aquellos vídeos que generan un mayor número de visualizaciones y de interacciones

Los booktubers seleccionados a partir de los datos proporcionados por Marquina (2016) son: (Tabla 1)

Para analizar la estructura se ha diseñado una ficha capaz de recoger datos de primera mano a través de la navegación en cada uno de los 
Tabla 1. Clasificación booktubers en base número de seguidores

\begin{tabular}{|c|c|}
\hline Denominación booktubers & Seguidores* \\
1. El coleccionista de mundos & 190.763 \\
2. Javier Ruescas & 170.805 \\
3. Fly like a butterfly & 165.674 \\
4. Andreo Rowling & 112.500 \\
5. May R Ayamonte & 90.287 \\
6. LibrosPorLeer & 76.728 \\
7. Never be Hopeless & 74.998 \\
8. Nube de palabras & 73.944 \\
9. MartitaraBookVlogs & 58.092 \\
10. Little Red Read & 42.235 \\
\hline
\end{tabular}

Cifras de seguidores enero 2017

perfiles. La aplicación permite conocer la fecha de creación del canal, las redes que tienen enlazadas y el número de suscriptores.

El análisis de contenido busca lograr encontrar el sentido latente que tiene el vídeo con mayor número de visualizaciones. Este análisis busca desvelar lo que subyace y lo inédito del mensaje (Raigada, 2002). (Tabla 2)

Tabla 2. Ficha análisis contenido

\begin{tabular}{|c|c|}
\multicolumn{2}{|c|}{ Ficha técnica } \\
\hline Fecha creación del canal & \\
\hline Redes sociales asociadas & \\
\hline Número de seguidores & \\
\hline Número más alto de visualizaciones & \\
\hline Contenido de lo más visitado & \\
\hline Número de interacciones & \\
\hline
\end{tabular}

\section{RESULTADOS}

El análisis de la estructura de contenidos de los casos analizados manifiesta una gran convergencia en el número de secciones creadas y en cómo son denominadas. Destaca la utili- zación, en casi todos los casos, de anglicismos para clasificarlas. A continuación, describimos las secciones y lo que aglutinan bajo su denominación:

- TBR o "to be read": piezas de corta duración en la que anuncian lo que van a leer.

- "Book hauls": contenido que se realiza cada fin de mes sobre los libros leídos en ese periodo con una breve explicación de porqué lo compraron.

- Reseña libros: los booktubers vierten su opinión sobre un libro, lo que más les ha gustado o lo que menos, siempre con un tono informal.

- "Book tags": bajo esta denominación desarrollan preguntas para sugerir libros que tratan una misma temática.

- "Wrap up": en este caso desarrollan piezas de corta duración donde comentan la experiencia con los libros leídos.

- "Unboxing": con estas piezas buscan crear expectación ya que el contenido gira entorno a mostrar el libro que han adquirido sin abrirlo.

- Sección preguntas y respuestas: son videos en donde responden a preguntas inquietudes que les hacen llegar sus seguidores.

- "Book self tours": es el video más esporádico ya que el contenido gira entorno a mostrar las bibliotecas con los libros comprados a lo largo de los meses.

El análisis del canal del booktuber tomando como referencia el contenido que más visualizaciones alcanza nos permite conocer qué temática y estructura del mensaje está resultando más atractiva. Para tal fin hemos desarrollado unas fichas que recogen el perfil del 
booktuber y los datos básicos de las piezas analizadas.

\subsection{CONTENIDO GENERALIS- TA}

El booktuber "El coleccionista de mundos" es el que ostenta en el momento de este análisis el mayor número de seguidores. La pieza que ha conseguido mayor número de visualizaciones en su canal no está relacionada con ninguna reseña de libro, sino que se centra en exponer lo que te reporta leer, es decir, los beneficios de la lectura desde un plano emocional. A través de un montaje sencillo en donde se suceden planos de recurso de bancos de imagen aparece un texto calado con mensajes cortos, de tono motivador e inspirador y acompañado por música de librería. La pieza apenas llega a los 4' de duración, lo que facilita su visualización.

Tabla 3. Ficha análisis contenido

\begin{tabular}{|c|c|}
\hline \multicolumn{2}{|c|}{ Ficha técnica de "El coleccionista de mundos" } \\
\hline Fecha creación del canal & 1 septiembre 2012 \\
\hline Redes sociales asociadas & $\begin{array}{c}\text { Facebook, Instagram, } \\
\text { Google+, Twitter }\end{array}$ \\
\hline $\begin{array}{c}\text { Seguidores } \\
\text { Número más alto de } \\
\text { visualizaciones }\end{array}$ & $\begin{array}{c}206.545 \\
\text { ciones }\end{array}$ \\
\hline $\begin{array}{c}\text { Contenido de lo más visi- } \\
\text { tado }\end{array}$ & ¿Por qué leer? \\
\hline Número de interacciones & 372 comentarios \\
\hline
\end{tabular}

\subsection{CONTENIDO HUMORÍSTI- CO}

En el caso de Javier Ruescas, el video que más visualizaciones le ha reportado es de corte humorístico relacionado con un personaje literario, concretamente Harry Potter. Al video se suman otros booktubers a los que invita a co- nocer al final de la pieza. El contenido sigue la estela de videos de humor fácil que funcionan bien en la red con la habilidad de relacionarlo con un personaje del mundo literario y con una escena de la película de la saga de Harry Potter. Existen más piezas de esta factura en su canal que le han reportado tener un gran número de visualizaciones.

Tabla 4. Ficha análisis contenido

\begin{tabular}{|c|c|}
\hline Fecha creación del canal & 1 septiembre 2012 \\
\hline Redes sociales asociadas & $\begin{array}{c}\text { Facebook, Instagram, } \\
\text { Google+, Twitter }\end{array}$ \\
\hline Seguidores & 170.805 \\
\hline $\begin{array}{l}\text { Número más alto de } \\
\text { visualizaciones }\end{array}$ & 206.545 visualizaciones \\
\hline $\begin{array}{l}\text { Contenido de lo más vi- } \\
\text { sitado }\end{array}$ & $\begin{array}{c}\text { Reto de las grageas de } \\
\text { Harry Potter }\end{array}$ \\
\hline Número de interacciones & 356 comentarios \\
\hline
\end{tabular}

\subsection{CONTENIDO POR GÉNERO LITERARIO}

Dos son las booktubers que forman parte de esta clasificación: "Fly like a butterfly" y "Andreo Rowling". Mientras que la primera nos presenta un ranking de sus 10 libros preferidos de género romántico. La segunda se centra en la recomendación de la saga de género fantástico de Harry Potter de la que se declara fan activa. En ambos casos la factura de la pieza grabada es sencilla y se dirigen a su público en un lenguaje sencillo y cercanos propio de adolescentes/ jóvenes (Tabla 5)

\subsection{CONTENIDO SUGERENCIA POR SIMILITUD}

El último caso analizado se distingue del resto por no centrarse en ningún género sino por 
Tabla 5. Ficha análisis contenido

\begin{tabular}{|c|c|}
\hline \multicolumn{2}{|c|}{ Ficha técnica de "Fly like a butterfly" } \\
\hline Fecha creación del canal & 25 marzo 2010 \\
\hline Redes sociales asociadas & $\begin{array}{c}\text { Facebook, Instagram, } \\
\text { Google+, Twitter }\end{array}$ \\
\hline Seguidores & 165.674 \\
\hline Número más alto de & $\begin{array}{c}550.308 \text { visualiza- } \\
\text { ciones }\end{array}$ \\
\hline Contenido de lo más visitado & $\begin{array}{c}\text { Libros románticos } \\
\text { juveniles }\end{array}$ \\
\hline Número de interacciones & 667 comentarios \\
\hline
\end{tabular}

Tabla 6. Ficha análisis contenido

\begin{tabular}{|c|c|}
\hline \multicolumn{2}{|c|}{ Ficha técnica de "Andreo Rowling" } \\
\hline Fecha creación del canal & 14 octubre 2012 \\
\hline Redes sociales asociadas & $\begin{array}{l}\text { Facebook, Instagram, } \\
\text { Google+, Twitter }\end{array}$ \\
\hline Seguidores & 112.500 \\
\hline $\begin{array}{l}\text { Número más alto de } \\
\text { visualizaciones }\end{array}$ & 202.063 visualizaciones \\
\hline $\begin{array}{c}\text { Contenido de lo más } \\
\text { visitado }\end{array}$ & $\begin{array}{c}\text { Mi colección de Harry } \\
\text { Potter }\end{array}$ \\
\hline Número de interacciones & 589 comentarios \\
\hline
\end{tabular}

basar la recomendación en la "empatía" del libro. La booktuber habla a cámara y muestra el libro que le gustó y el que por temática se acerca este por similitud de trama y contenido. La crítica que hace difiere de las críticas literarias de prensa. Simplemente destaca los momentos que más le engancharon de la historia o de los personajes y siempre usando un lenguaje llano y sencillo.
Tabla 7. Ficha análisis contenido

\begin{tabular}{|c|c|}
\hline \multicolumn{2}{|c|}{ Ficha técnica de "May R Ayamonte" } \\
\hline Fecha creación del canal & 5 junio 2011 \\
\hline Redes sociales asociadas & $\begin{array}{l}\text { Facebook, Instagram, } \\
\text { Google+, Twitter }\end{array}$ \\
\hline Seguidores & 90.287 \\
\hline $\begin{array}{l}\text { Número más alto de } \\
\text { visualizaciones }\end{array}$ & 179.365 visualizaciones \\
\hline $\begin{array}{l}\text { Contenido de lo más } \\
\text { visitado }\end{array}$ & $\begin{array}{c}\text { Recomendación libros, } \\
\text { "si te gusta esto... te gus- } \\
\text { tará..." }\end{array}$ \\
\hline Número de interacciones & 480 comentarios \\
\hline
\end{tabular}

\section{DISCUSIÓN Y CONCLUSIONES}

Los resultados de este estudio inicial sobre el fenómeno incipiente del perfil del booktuber manifiestan la consolidación de este nuevo influencer en el universo de las redes sociales. Estos jóvenes lectores, lejos de constituirse en críticos literarios tal y como los conocemos en la actualidad, buscan transmitir la pasión que tienen por leer comentando los últimos títulos que han adquirido y dando una visión cercana y muy personal de la lectura realizada. Con una frecuencia de al menos un video a la semana comparten todo lo que leen, cuando lo leen y como lo leen.

En general, la oportunidad comunicativa que ofrece el canal de YouTube facilita que la generación de los contenidos sea fácil de compartir y de seguir. La estrategia de difusión que siguen estos booktubers para conseguir un mayor número de visualizaciones es la de compartir cada nuevo vídeo al mismo tiempo en las redes sociales enlazadas. Todos los casos analizados utilizan las siguientes plataformas: Facebook, Instagram, Twitter y Google+, siempre 
teniendo como base generadora del contenido la plataforma de YouTube.

La cifra de seguidores si bien dista de otros youtubers consolidados en esta gran red social, empieza a tener cifras interesantes de suscriptores y de visualizaciones de contenido. Algo que sin duda algunas editoriales están utilizando en el lanzamiento de algunos títulos. Pero que sobretodo, se tendrá que tener en cuenta por parte de la administración pública y, en concreto, por el Ministerio de Educación como vehículo clave a la hora de establecer su estrategia de comunicación en la necesidad de conseguir un cambio en la tendencia del hábito de lectura hacia el que se encamina el público más joven en la actualidad.

Si bien este estudio refleja el análisis de una primera fase exploratoria, ya se pueden extraer conclusiones sobre la clasificación de su árbol de contenidos claramente inspiradas en los booktubers anglosajones. El modelo parece asentado, pero contenidos de tono humorístico como el analizado en el caso de Javier Ruescas nos hace pensar en una estrategia de tono que, siguiendo la estela de otros contenidos de YouTube, se convierten en los más compartidos.

Por último, cabe destacar el tono de comunicación persuasivo que utilizan estos influencer. Su lenguaje basado en tono directo, sencillo, honesto y llano y siempre apasionado, se acerca e impacta al target de consumo que tiene la red social. Este tono, junto a la autenticidad que reflejan los comentarios que vuelcan sobre las obras literarias, seguramente les haga ganar credibilidad en su discurso y tenga su reflejo en el número de seguidores, aspecto que refrendan los estudios mencionados a lo largo del artículo.

\section{REFERENCIAS}

AIMC (Asociación Investigación Medios de Comunicación) (2015). Navegantes en la red. Recuperado de http://www.aimc.es/otros-estudios-trabajos/navegantes-la-red/

Bluemagazine, (2016). BookTube, también hay youtubers que triunfan (y mucho) hablando de libros. (n.d.). Recuperado de http://www.bluebbva. com/2016/06/booktube-tambien-hay-youtubers-que-triunfan-y-mucho-hablando-de-libros.asp

Cordón García, J.A, (2016). La investigación sobre lectura en el entorno digital. Métodos de Información, [en línea] 7(13), pp.247-268. doi:10.5557/IIMEI7-N13-247268

Domínguez Correa, B. (2016) Análisis del fenómeno de BookTube en España. (Tesis Doctoral. Universidad Politécnica de Valencia, Valencia). Recuperado de http://hdl.handle.net/10251/75319 
Elite Daily and Millennial Branding (2015) Millennial Consumer Study. Recuperado de https://www.elitedaily.com/

Golfields Rodrigues, B. (2015, julio 15). Amazing BookTube Channels to Suit Every Reading Personality. Recuperado de http://www.huffingtonpost .ca/2015/03/24/booktube-channels_n_6933226.html

IAB (Internet Advertising Bureau) (2015). VI estudio de redes sociales de IAB Spain. Recuperado de http://www.iabspain.net/wpcontent/ uploads/ downloads/2015/01/Estudio_Anual_Redes_Sociales_2015. pdf

Katz, E., \& Lazarsfeld, P. F. (1966). Personal Influence, the part played by people in the flow of mass communications. Transaction Publishers.

Marquina, J. (2016). Los 10 booktubers españoles con mayor número de seguidores. Recuperado de http://www.julianmarquina.es/los-10-booktubers-espanoles-con-mayor-numero-de-seguidores/

Mason, W. A., Conrey, F. R., \& Smith, E. R. (2007). Situating social influence processes: Dynamic, multidirectional flows of influence within social networks. Personality and social psychology review, 11(3), 279-300.

MECD (Ministerio de Educación Cultura y Deporte) (2015). Informe sobre la lectura en España. Recuperado de http://www.mecd.gob.es/

ONTSI (Observatorio Nacional de las Telecomunicaciones y de la Sociedad de la Información) (2015). La sociedad en red. Recuperado de http://www.ontsi.red.es/ontsi/sites/ontsi/files/informe_anual_la_sociedad_en_red_2014_edicion_2015.pdf

Pindado, J. (2003). El papel de los medios de comunicación en la socialización de los adolescentes de Málaga. (Tesis doctoral. Universidad de Málaga, Málaga). Consultado de http://mc142.uib.es/

Raigada, J. L. P. (2002). Epistemología, metodología y técnicas del análisis de contenido. Sociolinguistic Studies, 3(1), 1-42.

Ravettino Destefanis, A. J. (noviembre, 2015). Booktubers y performances virtuales: modos contemporáneos de difundir y compartir literatura juvenil en la Red. Ponencia presentada en VIII Jornadas de Jóvenes Investigadores. Eje 4: Producciones, consumos y políticas estético-culturales. Nuevas tecnologías. Buenos Aires, Argentina.

The Cocktail Analysis (2015). VI Observatorio de Redes sociales. Recuperado de http://tcanalysis.com/blog/posts/vii-observatorio-redes-sociales

Urresti, M. (2008). Ciberculturas juveniles. Buenos Aires: La Crujía.

Vanderdorpe, C., 2011. Some considerations about the future of Reading. Digital Studies/le Champ Numerique, 2(2).

YouTube para la prensa. (2017, diciembre 04). (n.d.). Recuperado de https:// www.youtube.com/yt/press/es-419/statistics.html 